\title{
MACROSCOPIC GLOBAL MODELING OF BINARY ALLOY SOLIDIFICATION PROCESSES*
}

\author{
BY \\ V. ALEXIADES (Oak Ridge National Laboratory and University of Tennessee) \\ BY \\ D. G. WILSON AND A. D. SOLOMON (Oak Ridge National Laboratory)
}

\begin{abstract}
A macroscopic mathematical model is constructed describing the evolution of the phases of a binary alloy or mixture undergoing solidification under the action of simultaneous conduction of heat and diffusion of solute. The formulation is global, in the form of a pair of conservation laws valid over the whole region occupied by the alloy in a weak (distributional) sense. Thus it is especially convenient for numerical solution since it does not require tracking of the interface, which, in fact, may develop into a "mushy zone".
\end{abstract}

Introduction. The solidification of impure materials, such as alloys or mixtures, depends not only on the removal of sensible and latent heats but also on the redistribution of the impurity or solute. It is, therefore, a coupled heat and mass transfer process with the strongest coupling occuring at the solid-liquid interface. The freezing temperature is no longer constant but it depends on the concentration as dictated by the phase diagram of the alloy.

Qualitatively, such processes are fairly well understood at the macroscopic level, at least as far as what basic underlying phenomena are involved (cf. Chalmers [4]). At the microscopic level, significant advances in our understanding of solidification and other phase transformations have been achieved recently by the Theory of Critical Phenomena, a field of Physics experiencing tremendous growth lately.

Quantitatively, however the situation is very unsatisfactory as there is very little mathematical theory available. In view of the importance of solidification processes in today's science and technology, the quantitative understanding of such processes becomes imperative.

\footnotetext{
*Received November 15, 1983. Research sponsored by the Applied Mathematics Sciences Research Program, Office of Energy Research, U.S. Department of Energy under contract W-7405-eng-26 with the Union Carbide Corporation.
} 
The simplest coupled macroscopic mathematical model of binary alloy solidification consists of heat conduction and mass diffusion equations in the solid and in the liquid, together with interface conditions expressing energy and mass conservation. A fundamental assumption in the construction of such a model is that the solid-liquid interface is a smooth surface, just like in the solidification of a pure material. Such an assumption however may be physically unrealistic and may lead to self-inconsistency of the model as was observed in Wilson-Solomon-Alexiades [23] (cf. Sec. 2). To overcome such objections we construct here a new, more comprehensive, mathematical model of the binary alloy solidification process directly based on fundamental principles of modern (nonequilibrium) thermodynamics.

The model describes the time evolution of the phases of a binary alloy or mixture undergoing solidification under the action of simultaneous conduction of heat and diffusion of solute. It is general enough to allow thermal-diffusive cross effects (Soret and Dufour effects), as well as anisotropic thermodynamic and transport coefficients, which may depend on both concentration and temperature. The only physically restrictive assumptions we make are those of a constant density and of a monotonic phase diagram (as in Fig. 1). Both of these will be relaxed in future refinements of the model.

An important characteristic of our model is its global formulation, as a pair of conservation laws valid over the whole region occupied by the alloy in a weak (distributional) sense. The phases are distinguished only by the values of the quantity "liquid fraction" which is $\equiv 1$ in the liquid, $\equiv 0$ in the solid, and between 0 and 1 in the interface which may be a surface, a mushy zone or a very irregular set. The point here is that we make no a priori assumptions about the geometry of the interface, the system itself will develop it as it pleases (see, however, Trivedi [22]). At the same time, this makes the model especially convenient for numerical solution as no tracking of the interface is required. Numerical experiments will be reported separately. (Solomon-Alexiades-Wilson [19]).

In Sec. 1, a brief qualitative description of the alloy solidification process is given. An overview of existing approaches appears in Sec. 2 and some of the shortcomings are mentioned. The development of the model is described step-by-step in Sec. 3. The resulting system may fail to be parabolic and conditions for its parabolicity are obtained in Sec. 4. Finally, some important particular cases are noted in Sec. 4, such as the reduction of our model to the classical Stefan problem for the solidification of a pure material.

1. Qualitative description of binary alloy solidification. Consider a binary alloy or mixture consisting of components $A$ and $B$. The equilibrium phase diagram of the alloy describes the concentrations of liquid and solid that can co-exist in thermodynamic equilibrium as functions of temperature. The simplest such diagram, for an alloy capable of forming solid solutions in all proportions (such as $\mathrm{Cu}-\mathrm{Ni}$ ), is shown in Fig. 1 (cf. Chalmers [4, p. 5]).

As concentration variable $C$ we can use the mass fraction of component $B$; thus the concentration of $A$ is $1-C$. The zone between the liquidus and solidus curves represents states for which neither of the two pure phases is stable alone. Instead, both solid and 
liquid appear, each with the appropriate solidus $C^{s}$ and liquidus $C^{\prime}$ concentrations as determined by the temperature, $T$.

In a macroscopic time scale such that local thermodynamic equilibrium can be assumed to hold, the phase diagram determines the phase locally in terms of the concentration $C(x, t)$ and temperature $T(x, t)$ of an elementary cell located at $x$ at time $t$. Let us follow such a cell $\mathscr{P}$ which is initially liquid. As its temperature is lowered, the state of $\mathscr{P}$ reaches the liquidus curve at some point $\left(C^{l}, T\right)$ on the phase diagram (cf. Fig. 1). With further cooling, the first solid that appears has concentration $C^{s}>C^{\prime}$. Thus the liquid fraction of $\mathscr{P}$ is depleted of solute $B$ (or enriched in $A$ ) and its state goes down the liquidus (it will freeze at a lower temperature) while the state of the solid fraction of $\mathscr{P}$ goes down the solidus curve. The liquid fraction now appears supercooled (and the solid superheated). This is referred to as constitutional supercooling [4]. The average state of $\mathscr{P}$ now lies in the intermediate zone, which we shall call the inter-phase (or mushy) zone. This continues until all of $\mathscr{P}$ has solidified.

This is, of course, a very rough description of the process. The latent heat of fusion must be removed and the solute must be redistributed in a cooperative manner for solidification to occur. Thus the process involves the strong coupling of heat conduction, material diffusion and fluid flow, which makes the quantitative problem intractable.

2. Overview of existing approaches. The common practice is to limit attention to cases in which only one process dominates (usually diffusion or conduction, but not fluid flow) so that the other two can be ignored.

The most common approach probably is to ignore heat conduction and consider the temperature at a steady-state since conduction is usually much faster than diffusion. Another one is the case of a "well-stirred" liquid (cf. Alexiades [1]) used, for example, in the steel industry in connection with "dunking" of cold solid steel into liquid steel for temperature control. Alternatively, the effect of variable freezing temperature may be

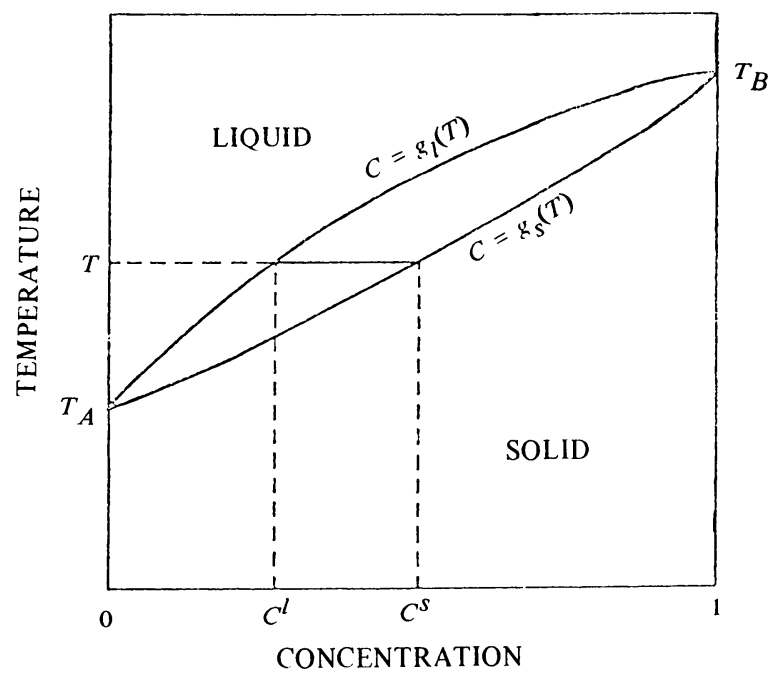

FIG. 1. An Equilibrium Phase Diagram. 
simulated by a mushy zone as in Tien-Geiger [21], Cho-Sunderland [5], Alexiades-Cannon [2], and Clyne [25].

The purpose of such simplifications is of course to avoid the coupling of the heat and mass transfer processes. Even then, the problem is not simple. The location of the solid-liquid interface is an unknown. This makes the problem an, inherently non-linear, moving boundary problem. One of the most extensively studied moving boundary problems is the so-called Stefan problem (Rubinstein [17]), which models the solidification of a pure material. The unknown quantities are the distribution of temperature and the location of the interface (a surface separating the solid and liquid). The determining conditions are the heat conduction equations in the solid and the liquid and a condition expressing energy conservation across the interface (the so-called Stefan condition), together with appropriate initial and boundary conditions. In this model it is assumed that heat conduction dominates (convection in the liquid is negligible). Classical solvability of the Stefan problem has been established only in the case of one space dimension (Fasano-Primicerio [9], Wilson-Solomon-Boggs [25]). In higher dimensions the only successful approach has been via weak formulations, which view the problem as a global conservation law and which are in fact closer to the physics of the problem (Elliot-Ockendon [8]). Such formulations, being of fixed-domain type, are especially convenient for numerical computations since they do not require tracking of the moving boundary. Recent work of Lacey and Shillor [12] also supports the contention that weak formulations may be the only reasonable ones in the presence of supercooling.

A mathematical model of binary alloy solidification has been suggested by Rubinstein [17] (see also Tayler [20]) as a generalization of the Stefan problem. In addition to the heat conduction equations for the temperature in the solid and liquid and the Stefan condition on the interface, solute diffusion equations for the concentration are also imposed as well as a corresponding condition expressing mass conservation across the interface. Moreover, the solidus and liquidus concentrations are related to the interface temperature via the phase diagram relations. Thus, the heat and mass transfer processes are coupled only at the interface, which is assumed to be a smooth surface. For a semi-infinite, one-dimensional material, the model admits a similarity solution for all nonnegative values of the physical parameters (assumed constants) (Rubinstein [17], Solomon-Wilson-Alexiades [18]). In this solution, however, the concentration distribution in the solid remains constant (even though the diffusivity there may be nonzero).

Numerical approximations for essentially the same model have been discussed by Fix [10], Crowley-Ockendon [7], Meyer [14], Bermudez-Saguez [3], Wilson-Solomon-Alexiades [24]. The appearance of a "mushy" zone was noted for some values of the parameters which could not be entirely attributed to numerical errors. Finally, we observed in [23] that, in fact, even the explicit solution of Rubinstein produces such a mushy zone when the material diffusivity in the liquid is small enough. This of course contradicts the assumption of a sharp interface on which the model itself is based and renders the model self-contradictory for certain ranges of parameter values. At the same time, it suggests that a good model should allow for the occurrence of a mushy zone, exhibiting the built-in advantages of weak formulations over the classical ones. 
Considerations of the stability of a sharp planar interface in the presence of constitutional supercooling (Langer [13], Christian [6]) also lead one to reject the classical Rubinstein model (cf. Lacey-Ockendon-Tayler [11]). In [11] an attempt is made to model the microstructure of the mushy zone and the difficulties this entails are discussed. ${ }^{1}$

3. The new model. Our mathematical formulation of the alloy solidification prrocess is based directly on the basic conservation laws of mass and internal energy, and on the assumption of local thermodynamic equilibrium (so that the phase diagram determines the phase locally at each instant of time). We model the process at a macroscopic level, entirely avoiding any discussion regarding the microstructure of the interface. At this stage we have omitted convective effects and chemical reactions, focusing only on the coupling between heat conduction and material diffusion. The mass and energy conservation equations are taken to hold globally over the region occupied by the alloy but in a distributional sense instead of pointwise in each phase separately (which is the case in Rubinstein's model described in Sec. 2). In other words, we begin with a "weak formulation" which reduces to a classical pointwise one whenever the functions involved are sufficiently smooth. The interface conditions are then automatically satisfied, provided that the interface is a smooth surface. Our weak formulation still expresses the fundamental conservation laws even if the interface is a thick region and not a surface.

3.1. General one-fluid equations of a binary mixture. We begin with general equations describing a compound fluid consisting of two components $A$ and $B$, always considered to be at the same temperature. We assume that there are no body forces acting, no chemical reactions and no convection taking place, so mass is transfered only by diffusion and heat only by conduction. Then the conservation laws for mass and energy are (cf. Woods [26, pp. 209-213]):

$$
\begin{gathered}
\rho \frac{\partial C_{i}}{\partial t}+\nabla \cdot \overrightarrow{J_{i}}=0, \quad i=A, B, \\
\rho \frac{\partial u}{\partial t}+\nabla \cdot \vec{q}=\phi,
\end{gathered}
$$

where $\rho \equiv$ constant is the fluid density, $C_{i}$ the concentration (mass fraction) of component $i, \vec{J}_{i}$ the diffusion momentum (= $\rho \vec{w}_{i}$, with $\vec{w}_{i}$ the diffusion velocity) of component $i, u$ the (thermodynamic) specific internal energy of the fluid mixture, $\vec{q}$ the energy flux and $\phi$ the internal heat source or sink (radiation heat). The only approximation made in the derivation of (3.2) is the "weak diffusion approximation" by which terms quadratic in the diffusion vectors $\vec{w}_{i}$ and $\vec{J}_{i}$ are neglected (Woods [26, p. 212]) and it follows that

$$
\vec{q}=\vec{Q}+\bar{h}_{A} \vec{J}_{A}+\bar{h}_{B} \vec{J}_{B},
$$

where $\vec{Q}$ denotes the heat flux and $\bar{h}_{i}$ the partial specific enthalpy of component $i$.

\footnotetext{
${ }^{1}$ After the circulation of a previous version of this work as an Oak Ridge National Laboratory Report, the recent paper by Luckhaus and Visintin [28] was brought to our attention by Professor Ockendon. Their appraoch is also via non-equilibrium thermodynamics but with a different aim. In the solid and liquid their model coincides with ours, but they assume that the interphase is a smooth surface. Thus they do not model the mushy zone, which is the main objective of this work.
} 
Constitutive equations, relating the fluxes $\vec{J}_{i}$ and $\vec{Q}$ to the thermodynamic driving forces $\nabla \bar{g}_{i}$ and $\nabla T / T$, where $\bar{g}_{i}=$ partial specific Gibbs energy (chemical potential) of $i$, are provided by the following first-order phenomenological laws of modern (irreversible) thermodynamics:

$$
\begin{gathered}
\vec{J}_{i}=-\sum_{j} \Lambda_{i j} \cdot \nabla\left[\bar{g}_{i}\right]_{T}+\delta_{i} \cdot \frac{\nabla T}{T}, \quad i=A, B, \\
\vec{Q}=-\kappa \cdot \frac{\nabla T}{T}+\sum_{j} \eta_{j} \cdot \nabla\left[\bar{g}_{j}\right]_{T} .
\end{gathered}
$$

Here $\left[\bar{g}_{i}\right]_{T}$ means we take $\bar{g}_{i}$ as if isothermal, the thermal contribution being included in the $\nabla T$ terms (cf. Rosenberger [16, p. 222]). They are generalizations of Fick's and Fourier's Laws (Woods [26, p. 215]). The phenomenological tensors $\kappa, \Lambda_{i j}, \delta_{i}, \eta_{i}$ have all the same structure (described in Woods [26, p. 215]) and satisfy Onsanger's reciprocal relations from which it follows that

$$
\begin{gathered}
\Lambda_{A A}=-\Lambda_{B A}=-\Lambda_{A B}=\Lambda_{B B}, \\
\delta_{A}=\eta_{A}=-\eta_{B}=-\delta_{B} .
\end{gathered}
$$

Since, also for a binary mixture, $\vec{J}_{A}+\vec{J}_{B}=\overrightarrow{0}$, we can express (3.3) and (3.4) as $\vec{J}_{A}=-\vec{J}_{B}$,

$$
\begin{gathered}
\vec{J}_{B}=-\Lambda_{B B} \cdot \nabla\left[\bar{g}_{B}-\bar{g}_{A}\right]_{T}+\boldsymbol{\delta}_{B} \cdot \frac{\nabla T}{T}, \\
\vec{Q}=-\kappa \cdot \frac{\nabla T}{T}+\boldsymbol{\delta}_{B} \cdot \nabla\left[\bar{g}_{B}-\bar{g}_{A}\right]_{T}, \\
\vec{q}=\vec{Q}+\left(\bar{h}_{E}-\bar{h}_{A}\right) \vec{J}_{B} .
\end{gathered}
$$

In addition to the conservation laws and constitutive relations, the Gibbs relation: $d u=T d s+\left(\bar{g}_{B}-\bar{g}_{A}\right) d C$ must hold and equations of state $u=u(C, T), \bar{g}_{i}=\bar{g}_{i}(C, T)$ must be specified.

Now we introluce the notations

$$
\begin{array}{ll}
\mu(C, T)=\bar{g}_{B}-\bar{g}_{A}, & (J / g) \\
\bar{h}(C, T)=\bar{h}_{B}-\bar{h}_{A}, & (J / g)
\end{array}
$$

and the following macroscopic transport coefficients:

$$
\begin{array}{ll}
\text { conductivity } \mathbf{k}(C, T)=\frac{1}{T} \boldsymbol{k} & \left(\mathrm{J} / \mathrm{m} \text {-sec }{ }^{\circ} C\right), \\
\text { diffusivity } \mathbf{D}(C, T)=\frac{1}{\rho} \frac{\partial \mu}{\partial C} \Lambda_{B B} & \left(\mathrm{~m}^{2} / \mathrm{sec}\right), \\
\text { thermal diffusion coefficient (Soret) } \boldsymbol{\delta}(C, T)=\frac{1}{T \rho} \boldsymbol{\delta}_{B} & \left(\mathrm{~m}^{2} / \mathrm{sec}{ }^{\circ} C\right), \\
\text { diffusion-thermo coefficient (Dufour) } \boldsymbol{\beta}(C, T)=\frac{\partial \mu}{\partial C} \boldsymbol{\delta}_{B} & (\mathrm{~J} / \mathrm{m} \mathrm{sec}) .
\end{array}
$$

All these tensors retain the structure of the original phenomenological ones [26, p. 215]. 
This notation already indicates that we consider the concentration $C$ and temperature $T$ as the primary independent thermodynamic variables describing the state of the material. In order to avoid confusion with the standard notation in which the internal energy is considered as a function of the entropy and concentration, we shall denote the specific energy $u$ by $e(C, T)$, (usually called enthalpy in the context of the Stefan problem). In terms of these independent variables the Gibbs relation becomes (see Appendix)

$$
d e=c d T+\left[\mu-T \frac{\partial \mu}{\partial T}\right] d C
$$

where $c(C, T)$ denotes the specific heat capacity of the fluid.

In this notation, the conduction-diffusion model for the binary fluid takes the following form:

Equations:

$$
\begin{gathered}
\frac{\partial C}{\partial t}=\nabla \cdot[\mathbf{D} \cdot \nabla C]-\nabla \cdot[\boldsymbol{\delta} \cdot \nabla T] \\
\rho \frac{\partial e}{\partial t}=\nabla \cdot[(\mathbf{k}-\rho \bar{h} \boldsymbol{\delta}) \cdot \nabla T]+\nabla \cdot[(\rho \bar{h} \mathbf{D}-\boldsymbol{\beta}) \cdot \nabla C]+\phi .
\end{gathered}
$$

Fluxes:

$$
\begin{aligned}
\vec{J}_{B} & =-\rho \mathbf{D} \cdot \nabla C+\rho \delta \cdot \nabla T, \\
\vec{Q} & =-\mathbf{k} \cdot \nabla T+\boldsymbol{\beta} \cdot \nabla C, \\
\vec{q} & =\vec{Q}+\vec{h}_{B} .
\end{aligned}
$$

Relations:

$$
\begin{aligned}
& d e=c d T+\left[\mu-T \frac{\partial \mu}{\partial T}\right] d C, \\
& e=e(C, T), \\
& \mu=\mu(C, T), \\
& \text { phase diagram. }
\end{aligned}
$$

3.2. The Gibbs relation. In the previous section nothing was said about the phase change process. The Eqs. (3.6)-(3.8) describe heat and mass transfer in any binary fluid, in any phase. The phases can only be distinguished by the equation of state

$$
e=e(C, T) \text {, }
$$

which we have to specify subject to the phase diagram and the Gibbs relation.

Our assumption of local thermodynamic equilibrium implies the validity of the equilibrium phase diagram of the alloy for the elementary cell $\mathscr{P}$. We consider $\mathscr{P}$ to be small enough to possess nearly uniform properties but much larger than a mean free path. The observer's time scale is taken to be of the order of magnitude of the intrinsic time scale $d t$ of $\mathscr{P}$ (the time it takes for an infinitesimal change in the state of $\mathscr{P}$ to occur).

We shall assume that the phase diagram of the alloy has the simple form shown in Figure 1. The liquids and solidus curves $T=f_{j}(C)$ or $C=g_{j}(T), j=l, s$, demarkate the 
three possible phases for $\mathscr{P}$ :

liquid, $\mathscr{P}_{l}: T \geqslant f_{l}(C)$ or $C \leqslant g_{l}(T)$,

solid, $\mathscr{P}_{s}: T \leqslant f_{s}(C)$ or $C \geqslant g_{s}(T)$,

inter-phase, $\mathscr{P}_{m}: f_{s}(C)<T<f_{l}(C)$ or $g_{l}(T)<C<g_{s}(T)$.

The inter-phase $\mathscr{P}_{m}$ is not a pure phase but a mixture of liquid and solid. Introducing the

$$
\text { liquid fraction } \lambda(C, T)=\left\{\begin{array}{lc}
1, & C \leqslant g_{l}(T) \text { (liquid) } \\
\frac{g_{s}(T)-C}{g_{s}(T)-g_{l}(T)}, & g_{l}(T)<C<g_{s}(T) \text { (inter-phase) } \\
0, & g_{s}(T) \leqslant C \text { (solid) }
\end{array}\right.
$$

by an application of the lever rule (cf. Chalmers [4, p. 6]), we can write any specific (extensive) property $X^{m}$ of $\mathscr{P}_{m}$ as

$$
X^{m}=\lambda X^{l}+(1-\lambda) X^{s}
$$

In the liquid or solid the Gibbs relation is

$$
d e^{j}=c_{j} d T+\left[\mu^{j}-T \frac{\partial \mu^{j}}{\partial T}\right] d C, \quad j=l, s .
$$

In fact, the term in the brackets coincides with the difference $\bar{h}^{j}:=\bar{h}_{B}^{j}-\bar{h}_{A}^{j}$ between the partial specific enthalpies at constant temperature, because

$$
\bar{h}=\mu-T \partial \mu / \partial T
$$

as we show in the Appendix (cf. (A7)). Hence

$$
d e^{j}=c_{j} d T+\bar{h}^{j} d C, \quad j=l, s .
$$

In the inter-phase, applying (3.10) to the energy $e$, we have

$$
e^{m}=\lambda e^{l}+(1-\lambda) e^{s},
$$

whence

$$
\begin{aligned}
d e^{m} & =\lambda d e^{l}+(1-\lambda) d e^{s}+\left(e^{l}-e^{s}\right) d \lambda \\
& =\left[\lambda \frac{\partial e^{l}}{\partial T}+(1-\lambda) \frac{\partial e^{s}}{\partial T}\right] d T+\left[\lambda \frac{\partial e^{l}}{\partial c}+(1-\lambda) \frac{\partial e^{s}}{\partial c}\right] d C+\left(e^{l}-e^{s}\right) d \lambda \\
& =\left[\lambda c_{l}+(1-\lambda) c_{s}\right] d T+\left[\lambda \bar{h}^{l}+(1-\lambda) \bar{h}^{s}\right] d C+\left(e^{l}-e^{s}\right) d \lambda .
\end{aligned}
$$

Note that

$$
L(T):=e^{l}\left(g_{l}(T), T\right)-e^{s}\left(g_{s}(T), T\right)=\text { latent heat }
$$

of fusion. Defining

$$
c_{m}=\lambda c_{1}+(1-\lambda) c_{s}
$$

and

$$
\bar{h}^{m}=\lambda \bar{h}^{l}+(1-\lambda) \bar{h}^{s},
$$


the Gibbs relation for $\mathscr{P}_{m}$ is

$$
d e^{m}=c_{m} d T+\bar{h}^{m} d C+L d \lambda
$$

In fact, since $d \lambda=0$ in the liquid or solid, the Gibbs relation (combining both (3.12) and (3.15))

$$
d e^{j}=c_{j} d T+\bar{h}^{j} d C+L d \lambda, \quad j=l, m, s,
$$

is valid in any phase $j=l, m, s$.

Note that chemical equilibrium between adjacent phases requires the chemical potential $\mu$ to be continuous across the inter-phase and therefore constant in $C$ at each $T$ in the inter-phase region of the phase diagram. Consequently, $\partial \mu / \partial C$ vanishes in the inter-phase and diffusion occurs there only due to the temperature dependence of the diffusivity $\mathbf{D}(C, T)$.

On the other hand, the specific heat, $c$, as well as the partial specific enthalpy, $\bar{h}$, are, in general, discontinuous across the inter-phase and the averagings (3.13) and (3.14) make the quantities

$$
c(C, T)=\left\{\begin{array}{lr}
c_{l}(C, T), & f_{l}(C) \leqslant T, \\
c_{m}(C, T), & f_{s}(C)<T<f_{l}(C), \\
c_{s}(C, T), & T \leqslant f_{s}(C),
\end{array}\right.
$$

and

$$
\bar{h}(C, T)=\left\{\begin{array}{lr}
\bar{h}^{\prime}(C, T), & f_{l}(C) \leqslant T, \\
\bar{h}^{m}(C, T), & f_{s}(C)<T<f_{l}(C), \\
\bar{h}^{s}(C, T), & T \leqslant f_{s}(C),
\end{array}\right.
$$

continuous but with discontinuous derivatives across the liquidus and solidus. Defining $e(C, T)$ similarly as $e^{l}, e^{m}, e^{s}$ according to phase, we can express the Gibbs relation (3.16) in any phase as

$$
d e=c d T+\bar{h} d C+L d \lambda
$$

3.3. Equation of state. The differential of energy is determined by the Gibbs relation (3.19). Since $e$ is a state variable, its value depends only on the initial and final states and not on the path joining these states on the phase diagram. Thus de must be an exact differential whenever smooth. This requirement imposes certain compatibility conditions among the thermodynamic data $c, \bar{h}, L$ and the phase diagram. Then we can define the energy $e(C, T)$ by integration of the exact differential de along a convenient path. Taking as reference state the point $\left(0, T_{r e f}\right)$, with $T_{r e f}$ a high temperature, and following the path shown in Fig. 2, the energy (drop) of $\mathscr{P}$ at state $(C, T)$ is given by the following 
expressions:

For $f_{l}(C) \leqslant T$,

$$
e(C, T)=\int_{0}^{C^{C}} \bar{h}^{\prime}\left(\xi, T_{\mathrm{ref}}\right) d \xi+\int_{T_{\mathrm{ref}}}^{T} c_{l}(C, \eta) d \eta ;
$$

For $f_{s}(C) \leqslant T \leqslant f_{l}(C)$,

$e(C, T)=\int_{0}^{C_{\bar{h}^{\prime}}}\left(\xi, T_{\mathrm{ref}}\right) d \xi+\int_{T_{\mathrm{ref}}}^{f_{l}(C)} c_{l}(C, \eta) d \eta+\int_{f_{l}(C)}^{T}\left[c_{m}(C, \eta)+L(\eta) \frac{\partial \lambda(C, \eta)}{\partial T}\right] d \eta$;

For $T \leqslant f_{s}(C)$,

$$
\begin{aligned}
e(C, T)= & \int_{0}^{C} \bar{h}^{l}\left(\xi, T_{\mathrm{ref}}\right) d \xi+\int_{T_{\mathrm{ref}}}^{f_{l}(C)} c_{l}(C, \eta) d \eta \\
& +\int_{f_{l}(C)}^{f_{s}(C)}\left[c_{m}(C, \eta)+L(\eta) \frac{\partial \lambda(C, \eta)}{\partial T}\right] d \eta+\int_{f_{s}(C)}^{T} c_{s}(C, \eta) d \eta .
\end{aligned}
$$

The term

$$
\int_{f_{l}(C)}^{T} L(\eta) \frac{\partial \lambda(C, \eta)}{\partial T} d \eta
$$

represents, of course, the latent heat contribution to the energy.

Introducing the augmented specific heat

$$
\hat{c}(C, T)=c(C, T)+L(T) \frac{\partial \lambda(C, T)}{\partial T},
$$

where $c$ is given by (3.17) and $\lambda$ by (3.9), we can write (3.20) simply as

$$
e(C, T)=\int_{0}^{C} \bar{h}^{\prime}\left(\xi, T_{\mathrm{ref}}\right) d \xi+\int_{T_{\mathrm{ref}}}^{T} \hat{c}(C, \eta) d \eta
$$

Thus $e(C, T)$ has discontinuous derivative $\partial e / \partial T=\hat{c}$, because $\partial \lambda / \partial T$ jumps across the liquidus and solidus curves. Note moreover that

$$
\frac{\partial \lambda(C, T)}{\partial T}=\frac{g_{s}^{\prime}(T)-\lambda\left[g_{s}^{\prime}(T)-g_{l}^{\prime}(T)\right]}{g_{s}(T)-g_{l}(T)}=\frac{\lambda g_{l}^{\prime}(T)+(1-\lambda) g_{s}^{\prime}(T)}{g_{s}(T)-g_{l}(T)}>0
$$

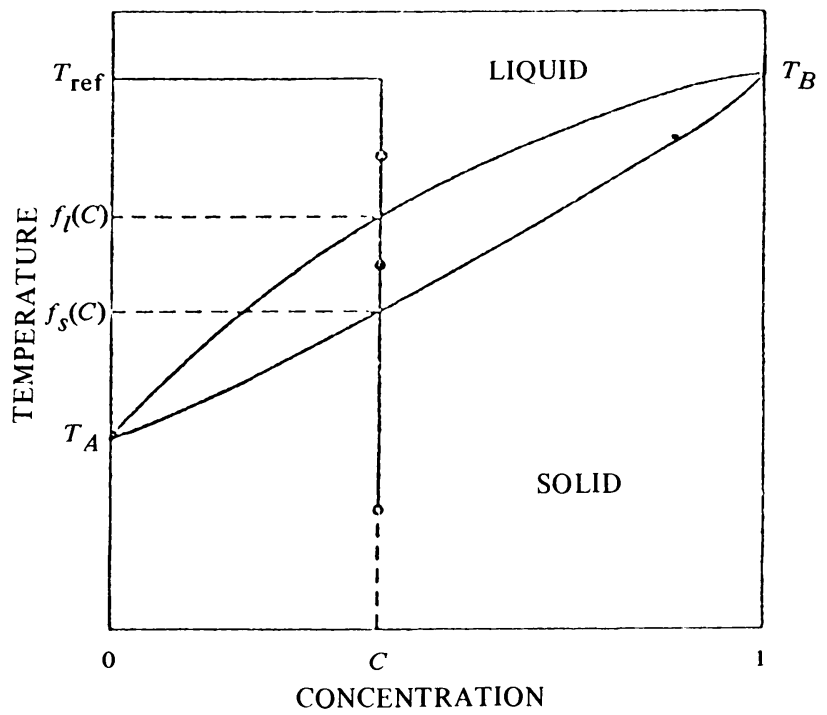

FIG. 2. Integration Path for Energy Computation. 
in the inter-phase since $g_{s}^{\prime}(T), g_{l}^{\prime}(T)>0$, and $\equiv 0$ in the liquid and solid. Hence

$$
\frac{\partial e}{\partial T}=\hat{c}(C, T)=c+L \frac{\partial \lambda}{\partial T} \geqslant c>0,
$$

(in fact it is bounded away from zero) everywhere, and therefore, given $C$ and $e$, the equation

$$
e(C, T)=e
$$

is uniquely solvable locally for $T$. It follows that the pair $C, e$ characterizes the state as well as $C, T$ does.

3.4. The binary alloy solidification model. In summary, the model consists of the Eqs. (3.6) with $e$ given by (3.22) (or more explicitly by (3.20)) together with the phase diagram.

The coupled PDE system (3.6) is to hold in the domain $\Omega$ occupied by the alloy. Since many of the coefficients, as well as $C, T$ and $e$ are not differentiable across interfaces, the PDEs can only be required to hold in distribution sense, as discussed earlier. Thus, they are valid pointwise wherever the variables are smooth and appropriate jump conditions will hold automatically. Such conditions could be stated explicitly if we assumed that an interface is a piecewise- $C^{1}$ surface (all one has to do is multiply the PDE's by a test function and integrate by parts separately over the liquid, inter-phase and solid). However, imposing the PDE's over the whole domain in distribution sense is both more general and more convenient, and also free of the shortcomings and inconsistencies of classical formulations.

The thermodynamic data $c, L, \mu$ as well as the transport coefficients $\mathbf{k}, \mathbf{D}, \boldsymbol{\delta}, \boldsymbol{\beta}$ are determined experimentally, at least in the liquid and solid. For theoretical purposes we assume them known everywhere and satisfying the necessary compatibility conditions for $e$ to be path-independent (cf. Sec. 3.3), and as we observed in Sec. 3.2 (see discussion following (3.16)) $\partial \mu / \partial C$ must vanish in the interphase. For practical purposes (computations), in order to fill the gaps in available data, one has to make physically reasonable approximations regarding their values.

The PDE system (3.6) is already in a form suitable for numerical solution. Knowing $C$, $T$ we can update $C$ and $e$ at the next time step and then use the definition of $e,(3.22)$, to find $T$. This is analogous to the "enthalpy method" for the Stefan problem. It requires however the inversion of the energy, i.e. solving for $T$ the complicated equation $e(C, T)=$ $e$. It is possible to write (3.6) in a more convenient form, by eliminating $e$ completely from the equations as follows.

We rewrite the Gibbs relation (3.19) in the form

$$
d e=\left[c+L \frac{\partial \lambda}{\partial T}\right] d T+\left[\bar{h}+L \frac{\partial \lambda}{\partial C}\right] d C,
$$

and use it to express $e_{t}$, in the energy equation, in terms of $T_{t}$ and $C_{t}$. We move the $C_{t}$-term to the right-hand-side and substitute for $C_{t}$ from the concentration equation. After some cancellations and regroupings, and using relation (3.11) the energy equation takes the form

$$
\begin{aligned}
\rho\left[c+L \frac{\partial \lambda}{\partial T}\right] T_{t}= & \nabla \cdot(\mathbf{k} \cdot \nabla T-\boldsymbol{\beta} \cdot \nabla C)+\rho L \frac{\partial(1-\lambda)}{\partial C} \nabla \cdot(\mathbf{C} \cdot \nabla C-\delta \cdot \nabla T) \\
& +\rho \nabla \bar{h} \cdot(\mathbf{C} \cdot \nabla C-\boldsymbol{\delta} \cdot \nabla T J)+\psi
\end{aligned}
$$


Let us point out that $\partial \lambda / \partial T$ and $\partial \lambda / \partial C$ are explicitly known from (3.9). In fact, $\partial \lambda / \partial T$ is given in (3.23) where we noted that it is always $\geqslant 0$. Similarly,

$$
\begin{aligned}
\frac{\partial(1-\lambda)}{\partial C}=-\frac{\partial \lambda}{\partial C}=+\frac{1}{g_{s}(T)-g_{l}(T)} & >0 \quad \text { in the inter-phase } \\
& \equiv 0 \quad \text { elsewhere. }
\end{aligned}
$$

In summary, the model consists of the strongly coupled quasilinear (not necessarily parabolic) system for the unknowns $C$ and $T$ :

$$
\begin{gathered}
C_{t}=\nabla \cdot(\mathbf{D} \cdot \nabla C-\boldsymbol{\delta} \cdot \nabla T), \\
\rho\left[c+L \frac{\partial \lambda}{\partial T}\right] T_{t}=\nabla \cdot(\mathbf{k} \cdot \nabla T-\beta \cdot \nabla C)+\rho L \frac{\partial(1-\lambda)}{\partial C} \nabla \cdot(\mathbf{D} \cdot \nabla C-\delta \cdot \nabla T) \\
+\nabla(\rho \bar{h}) \cdot(\mathbf{D} \cdot \nabla C-\delta \cdot \nabla T)+\psi
\end{gathered}
$$

and the phase diagram of the alloy as in Fig. 1. The system is to hold in distributional sense in the region $\Omega$ occupied by the alloy, and appropriate initial and boundary conditions must be specified.

\section{Parabolicity of the PDE System.}

4.1. Parabolicity condition. A system of partial differential equations of the form (3.25) is classified as parabolic if the coefficient matrix of the highest order derivatives is positive. For a $2 \times 2$ non-symmetric matrix $\left[a_{i j}\right]$ this amounts to the condition

$$
\left(a_{12}+a_{21}\right)^{2}<4 a_{11} a_{22} .
$$

Before we can apply this to our system its principal coefficients must have the same units, and the simplest way of achieving this is to undimensionalize the temperature by replacing $T$ by $T / \Delta T$ for some convenient temperature difference $\Delta T$ (e.g. $\Delta T=T_{B}-T_{A}$ ). The resulting condition for parabolicity is (for scalar $\mathbf{D}, \mathbf{k}, \boldsymbol{\delta}, \boldsymbol{\beta}$ )

$$
\left[\frac{\beta+\rho L D \lambda_{C}}{\rho \hat{c} \Delta T}+\delta \Delta T\right]^{2}<4 D \frac{k-\rho L \delta \lambda_{C}}{\rho \hat{c}} .
$$

4.2. Parabolicity in the absence of cross effects. For $\beta \equiv \delta \equiv 0$, the parabolicity condition (4.2) reduces to

$$
\left[\frac{L D \lambda_{C}}{\hat{c} \Delta T}\right]^{2}<4 D \frac{k}{\rho \hat{c}}
$$

which can be rearranged in the form

$$
\left[\frac{\partial \lambda}{\partial C}\right]^{2}<4\left(\frac{k \Delta T}{\rho D L}\right)\left(\frac{\hat{c} \Delta T}{L}\right)
$$

We observe immediately that in the solid and liquid where $\lambda_{C} \equiv 0$, the condition is satisfied automatically. In the inter-phase, we have

$$
\lambda_{C}=-\frac{1}{g_{s}(T)-g_{l}(T)} \quad \text { and } \quad \hat{c}=c+L \lambda_{T} \geqslant c
$$


(see (3.9) and (3.23)), so (4.3) will be satisfied if

$$
4\left(\frac{k \Delta T}{\rho D L}\right)\left(\frac{c \Delta T}{L}\right)\left[g_{s}(T)-g_{l}(T)\right]^{2}>1 .
$$

This can be expressed in a better form by introducing the thermal diffusivity $\alpha:=k / \rho c$. Then, the condition sufficient for parabolicity becomes

$$
4\left(\frac{\alpha}{D}\right)\left(\frac{c \Delta T}{L}\right)\left[g_{s}(T)-g_{l}(T)\right]^{2}>1 .
$$

Typically, $\alpha / D \gg 1$ and the Stefan number $c \Delta T / L>1$, so (4.4) can fail only for temperatures very close to $T_{A}$ (see (Fig. 1)). We conclude that

in the absence of cross effects, the system (3.25) can fail to be

parabolic only inside the inter-phase and only for tempera-

tures very close to $T_{A}$.

4.3. Parabolicity in the presence of cross effects. For simplicity we consider the situation only in the solid and liquid where $\lambda_{C} \equiv \lambda_{T} \equiv 0$. Then (4.2) reduces to

$$
\left[\frac{\beta}{\rho c \Delta T}+\delta \Delta T\right]^{2}<4 D \alpha
$$

where $\alpha=k / \rho c$. Since, typically, $\alpha>D$, simple sufficient conditions for parabolicity in this case are

$$
\frac{\beta}{\rho c \Delta T} \text { and } \delta \cdot \Delta T<D \leqslant \alpha .
$$

It is conceivable however that (4.5) (or rather (4.2)) may be violated for some materials. This will happen, if, for example,

$$
\beta>0, \quad \delta \cdot \Delta T>2 \alpha \geqslant 2 D,
$$

in which case the system will not be parabolic. We do not know if such materials exist.

\section{Particular cases of the general model.}

5.1. No cross effects. It is almost always the case that the coefficients $\delta$ and $\beta$, which measure the Soret and Dufour effects, are much smaller in magnitude than the direct effect coefficients $\mathbf{k}$ and $\mathbf{D}$. Thus, taking $\boldsymbol{\delta}=\boldsymbol{\beta}=\mathbf{0}$, the system becomes

$$
\begin{aligned}
& C_{t}=\nabla \cdot(\mathbf{D} \cdot \nabla C), \\
& \rho \hat{c} T_{t}=\nabla \cdot(\mathbf{k} \cdot \nabla T)+\rho L \frac{\partial(1-\lambda)}{\partial C} \nabla \cdot(\mathbf{D} \cdot \nabla C)+\nabla(\rho \bar{h}) \cdot(\mathbf{D} \cdot \nabla C)+\psi,
\end{aligned}
$$

where $\hat{c}=c+L \partial \lambda / \partial T$ (as in (3.20)).

5.2. Slowly varying chemical potential. If the chemical potential can be considered as constant during the process, then so is $\bar{h}$, so $\nabla \bar{h} \equiv 0$ and the temperature equation in (5.1) reduces to (also taking $\mathbf{k}, \mathbf{D}$ as scalars for simplicity):

$$
\rho\left[c+L \frac{\partial \lambda}{\partial T}\right] T_{t}=\nabla \cdot(k \nabla T)+\rho L \frac{\partial(1-\lambda)}{\partial C} \nabla \cdot(D \nabla C)+\phi .
$$


In the liquid or solid, this is simply the heat equation

$$
\rho c T_{t}=\nabla \cdot(k \nabla T)+\phi,
$$

but in the inter-phase it is augmented by two latent heat terms. One term affects the specific heat and the other adds a heat source due to material diffusion. The diffusion equation in (5.1) and (5.2) constitute a corrected version of the Rubinstein model [16], which does not a priori preclude the appearance of a mushy zone.

Note that taking $D=0$ in the inter-phase uncouples the equations and then (5.2) is exactly the conduction equation used in [2] and [27] to account for the latent heat evolution.

5.3. Recovery of the Stefan problem. For a pure material we have

$$
\mu \equiv 0, \quad C \equiv \text { constant }, \quad f_{l}(C)=f_{s}(C)=\text { const. }=T_{c r}, \quad g_{s}(T) \equiv g_{l}(T) .
$$

Thus, the liquid fraction becomes the Heaviside step function $H$ :

$$
\lambda(T)=\left\{\begin{array}{ll}
1, & T>T_{c r} \\
0, & T<T_{c r}
\end{array}=H\left(T-T_{c r}\right)\right.
$$

and the specific heat

$$
\hat{c}=c+L \frac{\partial \lambda}{\partial T}=c+L \cdot \delta\left(T-T_{c r}\right),
$$

where $\delta$ denotes the Dirac delta. Then, taking $T_{\text {ref }}=T_{c r}$ in (3.15),

$$
e(T)=\int_{T_{c r}}^{T} \hat{c}(\xi) d \xi=\int_{T_{c r}}^{T} c(\xi) d \xi+L \cdot H\left(T-T_{c r}\right)
$$

This is exactly the "enthalpy" of the classical Stefan Problem (cf. Elliot-Ockendon [8]). Our model reduces to

$$
\rho e_{t}=\nabla \cdot(k \nabla T)+\phi,
$$

which is the usual enthalpy formulation of the Stefan problem.

Appendix. We consider the temperature $T$ and concentration $C$ as the independent thermodynamic variables and seek to express the Gibbs relation

$$
d u(s, C)=T d s+\mu d C
$$

in terms of these variables. Recall that the pressure-volume term is missing due to our assumption of constant density.

Replacing $u(s, C)$ by $e(C, T)$, whence

$$
d e=\frac{\partial e}{\partial T} d T+\frac{\partial e}{\partial C} d c,
$$

and $d s$ by

$$
\frac{\partial s}{\partial T} d T+\frac{\partial s}{\partial C} d C,
$$


(A1) becomes

$$
d e=T \frac{\partial s}{\partial T} d T+\left[\mu+T \frac{\partial s}{\partial C}\right] d C
$$

and therefore we have

$$
\frac{\partial e}{\partial T}=T \frac{\partial s}{\partial T}=c(C, T)=\text { specific heat capacity },
$$

and

$$
\partial e / \partial C=\mu+T \partial s / \partial C .
$$

Differentiating (A3) w.r.t. $C$, (A4) w.r.t. $T$, and equating the results we see that

$$
\partial s / \partial C=-\partial \mu / \partial T .
$$

Therefore (A2) takes the form

$$
d e=c d T+[\mu-T \partial \mu / \partial T] d C .
$$

Next we show that

$$
\mu-T \partial \mu / \partial T=\bar{h}
$$

where $\bar{h}:=\bar{h}_{B}-\bar{h}_{A}$ with

$$
\bar{h}_{i}=\left(\partial h / \partial C_{i}\right)_{T, C_{j}}=\text { partial specific enthalpy of species } i=A, B \text {. }
$$

Indeed, the (specific) enthalpy $h$, Gibbs function $g$, entropy $s$ and temperature $T$ are related by (see Woods [26]) $h=g+T s$. Differentiating w.r.t. $C_{i}, i=A, B$ under constant temperature, subtracting the results and using (A5)

$$
\bar{h}=\bar{h}_{B}-\bar{h}_{A}=\bar{g}_{B}-\bar{g}_{A}+T\left(\frac{\partial s}{\partial C_{B}}-\frac{\partial s}{\partial C_{A}}\right)=\mu-T \frac{\partial \mu}{\partial T} .
$$

\section{REFERENCES}

[1] V. Alexiades, Rapid freezing of dilute allovs, IMA J. Applied Math., 30, 67-79 (1983)

[2] V. Alexiades and J. R., Cannon, Free boundary problems in alloy solidification, SIAM J. Math. Analysis, 11. 254-264 (1980)

[3] A. Bermudez and C. Saguez, Etude Numerique d' un Probleme de solidification d'un alliage, INRIA Report, 1981

[4] B. Chalmers, Principles of Solidification, Wiley, 1964

[5] S. H. Cho and J. E. Sunderland, Heat conduction problems with melting or freezing. J. Heat Transfer, 91C, 421-426 (1969)

[6] J. Christian, The theory of transformations in metals and alloys, Pergamon, Oxford, 1965

[7] A. B. Crowley and J. R. Ockendon, On the numerical solution of an alloy solidification problem, Int. J. Heat Mass Transfer, 22, 941-947 (1979)

[8] C. M. Elliot and J. R. Ockendon, Weak and variational methods for moving houndary problems, Pitman. Boston, 1982

[9] A. Fasano and M. Primicerio, General free boundary problems for the heat equation, I. II, III, J. Math. Analysis Appl. 57, 694-723 (1977); 56, 209-231 (1977); 59, 1-14 (1977)

[10] G. J. Fix, Numerical methods for alloy solidification problems, pp. 109-128 in [22]

[11] A. A. Lacey, J. R. Ockendon and A. B. Tayler, Modelling mushy regions, IMA J. Appl. Math. (to appear)

[12] A. A. Lacey and M. Shillor, The existence and stability of regions with super heating in the classical two-phase one-dimensional Stefan problems with heat sources, IMA J. Appl. Math. (to appear)

[13] J. S. Langer, Instabilities and pattern formation in crystal growth. Review Mod. Physics, 52, 1-28 (1980) 
[14] G. H. Meyer, A numerical method for the solidification of a binary alloy, Int. J. Heat Mass Transfer, 24, 778-781 (1981)

[15] J. R. Ockendon and W. R. Hodgkins, Moving boundary problems in heat flow and diffusion, Clarendon Press, Oxford, 1975

[16] F. Rosenberger, Fundamentals of crystal growth, I, Springer-Verlag, Berlin, 1979

[17] L. Rubinstein, The Stefan problem, AMS Transl. 27, Amer. Math. Society, Providence, 1971

[18] A. D. Solomon, D. G. Wilson and V. Alexiades, Explicit solutions to phase change problems, Quarterly of Appl. Math., 41 (1983)

[19] A. D. Solomon, D. G. Wilson and V. Alexiades, A numerical simulation of a binary alloy solidification process, SIAM J. Scient. Stat. Comp., to appear.

[20] A. B. Tayler, The mathematical formulation of Stefan problems, pp. 120-137 in [14].

[21] R. H. Tien and G. E. Geiger, $A$ heat transfer analysis of the solidification of a binary entectic system, J. Heat Transfer, 89C, 230-234 (1967)

[22] R. Trivedi, Theory of dendritic growth during the directional solidification of binary alloys, Journal of Crystal Growth, 49, 219-232 (1980)

[23] D. G. Wilson, A. D. Solomon and V. Alexiades, A shortcoming of the explicit solution for the binary alloy solidification problem, Letters in Heat and Mass Transfer, 9, 421-428 (1982)

[24] D. G. Wilson, A. D. Solomon and V. Alexiades, A model of binary alloy solidification, Int. J. Numer. Methods. Eng., 20(6), 1067-1085 (1984)

[25] D. G. Wilson, A. D. Solomon and P. T. Boggs, editors, Moving boundary problems, Academic Press, 1978

[26] M. Woods, Thermodynamics of fluid systems, Oxford Univ. Press, 1975

[27] T. W. Clyne, Numerical modeling of directional solidification of metallic alloys, Metal science 16, 441-450 (1982)

[28] S. Luckhaus and A. Visintin, Phase transition in multicomponent systems, Manuscripta Math. 43, 261-288 (1983) 